\title{
Surface EMG signal segmentation based on HMM modelling: Application on Parkinson's disease
}

\author{
Hichem Bengacemi, Abdenour Hacine Gharbi, Philippe Ravier, Karim Abed-Meraim, and Olivier Buttelli
}

\begin{abstract}
The study of burst electromyographic (EMG) activity periods during muscles contraction and relaxation is an important and challenging problem. It can find several applications like movement patterns analysis, human locomotion analysis and neuromuscular pathologies diagnosis such as Parkinson disease. This paper proposes a new frame work for detecting the onset (start) / offset (end) of burst EMG activity by segmenting the EMG signal in regions of muscle activity (AC) and non activity (NAC) using Discrete Wavelet Transform (DWT) for feature extraction and the Hidden Markov Models (HMM) for regions classification in AC and NAC classes. The objective of this work is to design an efficient segmentation system of EMG signals recorded from Parkinsonian group and control group (healthy). The results evaluated on ECOTECH project database using principally the Accuracy (Acc) and the error rate (Re) criterion show highest performance by using HMM models of 2 states associated with GMM of 3 Gaussians, combined with LWE (Log Wavelet decomposition based Energy) descriptor based on Coiflet wavelet mother with decomposition level of 4. A comparative study with state of the art methods shows the efficiency of our approach that reduces the mean error rate by a factor close to 2 for healthy subjects and close to 1.3 for Parkinsonian subjects.
\end{abstract}

Keywords-surface EMG signal, EMG signal segmentation, muscle activity, wavelet analysis, HMM models, Parkinson disease.

NOMENCLATURE

$\begin{array}{ll}\text { sEMG } & \text { surface Electromyographic. } \\ \text { HMM } & \text { Hidden Markov Modelling. } \\ \text { GMM } & \text { Gaussian Mixture Models. } \\ \text { DWT } & \text { Discrete Wavelet Transform. } \\ \text { DWE } & \begin{array}{l}\text { Discrete Wavelet decomposition } \\ \text { based calculus Energy. }\end{array} \\ & \text { Log Wavelet decomposition based Energy. } \\ \text { WCC } & \text { Wavelet Cepstral Coefficients. } \\ \text { LPC } & \text { Linear Prediction Coefficients. } \\ \text { HTK } & \text { Hidden Markov Model Toolkit. } \\ \text { NAC } & \text { No-Activity. } \\ \text { AC } & \text { Activity. } \\ \text { MUAP } & \text { Motor Unit Action Potentials. } \\ \text { STD } & \text { Standard Deviation. } \\ \text { ALE } & \text { Absolute Latency Error. } \\ \text { Acc } & \text { classification accuracy. } \\ \text { Re } & \text { Error Rate. }\end{array}$

Manuscript April 21, 2021; revised July 18, 2021.

Hichem Bengacemi is with the Signal Processing Lab, École Militaire Polytechnique, Algiers, Algeria (e-mail: bengacemihichem@yahoo.fr).

Abdenour Hacine Gharbi is with the LMSE lab, University of Bordj Bou Arreridj, Algeria. (e-mail: gharbi07@yahoo.fr).

Philippe Ravier, Karim Abed-Meraim and Olivier Buttelli are with the PRISME Laboratory, University of Orléans, 12 Rue de Blois, 45067, Orléans, France (e-mail: philippe.ravier@univ-orleans.fr, karim.abed-merim@univorleans.fr, olivier.buttelli@univ-orleans.fr)

Digital Object Identifier (DOI): 10.53907/enpesj.v1i1.27

\section{INTRODUCTION}

Muscle activity generates electric fields that can be picked up by means of electrodes located on the skin above the muscle under study. This signal and this technique are referred to as surface electromyographic activity (sEMG). Electromyography has been widely used to gain fundamental knowledge on neuromuscular control and muscle operating conditions (central and peripheral properties of this system, respectively) [1]. sEMG is also a relevant tool for the kinesiological analysis of movement disorders and for the evaluation of gait and posture such as for Parkinson's disease [2]. Accurate determination of the sEMG burst activation timing is an important consideration in motion analysis and remains a challenge and all the more so as sEMG recording are being done from patient with motor disorder. Timing features of the burst activation are the starting (onset) and the ending (offset) points which are relevant indicators to define motor coordination and its modifications during dynamic exercise such as during walking activity. This paper is dedicated to this key task, known in the literature under different narmes: signal segmentation or signal activity monitoring, or even burst detection.

\section{A. Related work}

The automatic methods for EMG signal segmentation can be divided into two categories. The first category covers the unsupervised methods like single and double threshold based methods [3, 4], statistical optimal decision-based methods [5-8], probabilistic-based methods [9] where the authors characterize the muscle activity using EMG burst presence probability (EBPP) estimated from HMM parameters, wavelet transformbased methods [10], clustering based methods [11], nonlinear energy operator-based methods [12-14] and adaptive energy method [15]. Performance of these methods considerably vary as they have different properties, computational complexities and tuning parameters. Moreover, all these methods are user- 
dependent since at least one of their tuning parameters must be set by the user for each considered sEMG signal.

The second category covers the supervised methods which learn from signals with segmentations (labels). In order to achieve the learning task, these methods need labelled dataset for the training phase. A testing dataset is used in a second phase for evaluating the performance of the method. The authors in [16] proposed to select the best one among a pool of onset estimators, given the measured features. The authors in [17] use Gaussian Mixture Models (GMM) clustering and Ant colony classifier (AntCC) as supervised method to automatize the processing of a large amount of sEMG signals. When applied to sEMG signals, these approaches clearly show improvement with respect to unsupervised methods. The proposed work falls within this category where we exploit the ECOTECH dataset [18] with Hidden Markov Modelling (HMM) to construct an automatic segmentation system for muscle activity monitoring.

\section{B. Our contribution}

The proposed system is based on HMM modelling combined with feature extraction based on Discrete Wavelet Transform (DWT). Several features descriptors extracted from discrete wavelet analysis have been studied such as Discrete Wavelet decomposition based calculus Energy (DWE), Log Wavelet decomposition based Energy (LWE) and Wavelet Cepstral Coefficients (WCC). The main task consists of looking for optimal parameters of HMM and wavelets descriptors to achieve the best EMG activity monitoring performance.

The HMMs have been widely investigated and employed in the automatic speech recognition. Recently, it has been successfully used for both medical monitoring and diagnosis system applications such as ECG classification [19], EEG classification [20], electrical appliances identification [21]. Especially, this method is also used for the PD classification using the raw gait data [22]. The HMM has been also combined with support vector machine (SVM) classifier for natural gesture recognition using EMG signals for upper limb prostheses control [23]. In [24], the authors have combined the HMM and the multilayer perceptrons (MLP's) for classifying six motions based on EMG signals. A HMM based classifier is used for speech recognition using myoelectric signals from the muscles of vocal articulation [25]. In [9], the authors have used the HMM on EMG signals to measure the EMG burst presence probability (EBPP). the study was limited to simulated signals and to one experimental signal just for illustration purpose. In this paper, we use HMM to classify EMG activity versus no EMG activity.

In brief, the main contributions of our work are: firstly, this work exploits the principal advantage of wavelet decomposition that is better adapted than Fourier decomposition for extracting the impulsive information of the action potentials (AP) of the motor units (MU). Secondly, this work adapts the HMM to automatic EMG signal segmentation. Note that HMM is one of the best tools to model signal state transitions and, to the best of our knowledge, this is the first time it is used for the considered supervised segmentation task. Finally, based on the ECOTECH data base, we provide a thorough performance analysis and comparative study with the state of the art methods.

The paper is organized as follows: section II. presents the prob- lem formulation and the proposed method. Section III. is dedicated to the performance analysis and discussions while section IV. is dedicated to the concluding remarks.

\section{MATERIAL AND Method}

In this section, we present in details the methodology of our proposed work. More precisely, after defining thoroughly the problem and work objectives, we introduce gradually the proposed method starting by the description of Hidden Markov Model (HMM) for EMG signal modelling. Then, the discrete wavelet transform and the proposed feature extraction technique are introduced. After that, we briefly describe the data base used in this study.

\section{A. Problem formulation of EMG signal segmentation}

We consider $N$ measurements (samples) $\{y[n]\}_{n=1: N}$ of surface EMG signal. Given a chosen analysis window length, these observations are divided into overlapping frames. For each signal's frame, we are interested in determining whether it contains a signal $s[n]$ embedded in a random background noise $w[n]$ (EMG activity) or, on the contrary, it is just the confusing manifestation of the noise (no EMG activity). Hence, we have a binary decision problem expressed as:

$$
\Gamma:\left\{\begin{array}{l}
H_{0}: \text { no EMG activity } \\
H_{1}: \text { EMG activity }
\end{array}\right.
$$

This task is known as EMG signal segmentation. In this work, the HMM method has been adapted to resolve the muscle activity detection problem or equivalently the EMG signal segmentation.

In addition, the visual inspection for activation tags (bursts onset and offset) was done by taking into account biomechanical indicators of the gait cycle calculated from accelerometer measurement. We were thus able to recalibrate the EMG activity with respect to the gait cycle. This is why the burst of activity defined in our recordings corresponded to a functional activation for walking and not to a non-voluntary activation.

\section{B. Hidden Markov Model (HMM) for EMG signal modelling}

Signal segmentation is the process of identifying the boundaries between segments of different classes. Signal segmentation system can be carried out using statistical modelling techniques such as Gaussian Mixture Models (GMM) and Hidden Markov models (HMM). Such system can be seen as pattern recognition system which requires a training phase for modelling the temporal pattern classes and recognition phase for identifying the different classes of segments of input signal. Hence, training and testing databases of signals are required to design the segmentation system. Both phases require feature extraction step to convert each signal in sequence of features vectors obtained by dividing the signal into overlapping windows and computing from each window a set of features that constitutes the feature vector (see Fig.1). This sequence of vectors can be considered as input sequence of observations in modelling or classification steps.

In this work, we used 50\% overlapping windows. 


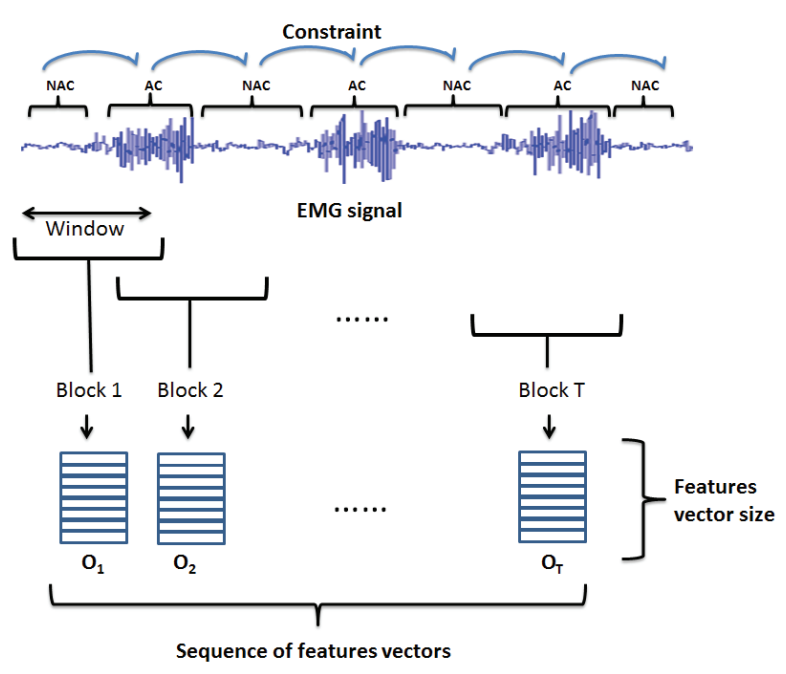

Fig. 1: Features extraction steps for HMM modelling. The blue arrows specify the authorized sequence of classes which defines constraints embedded in the language model for HMM design.

Particularly, in speech recognition task, the signal segmentation process has the aim to identifying the boundaries of segments of phonemes, word or sentences using respectively acoustic model, lexical model and language model. HMM models based system can be implemented using the HTK tools library (Hidden Markov Model Toolkit) which was designed firstly for speech recognition system including acoustic, lexical and language modelling [26]. This toolkit permits to model each phoneme by an HMM model of $N_{\text {states }}$ states each one associated to GMM model of $N_{G M M}$ Gaussian components for modelling the observation probability density function. The parameters of HMM models are estimated using the embedded Baum-Walsh algorithm which is implemented in HTK software using the HEREST command. The recognition phase can be carried out with the Viterbi algorithm using the HVITE command with respect to a language model and dictionary [26]. Nevertheless, the HMM models based system using HTK tools library has been used for others applications such as electrical appliance identification [27], speech recognition [28] and emotion recognition [29].

In this work, we use this toolkit to segment the EMG signal in regions (patterns) of activity (AC) class and regions of No-activity (NAC) class. The EMG signal is considered as a sequence of successive regions of AC and NAC classes, each one being represented by an observations sequence (sequence of features vectors) and modelled by HMM (see Fig.1)). The parameters of each model of AC class or NAC class are estimated from the training phase. By considering the same approach as for speech signal segmentation, the EMG signal segmentation uses the Viterbi algorithm to recognize the classes sequence using the trained HMM with respect to a language model that represents the constraint about the authorized sequence of classes.

1-HMM models: The HMM is a statistical model used to represent the evolution of temporal dynamics in time series or temporal observations sequence. The modelled phenomenon by HMM is a random and unobservable process that generates random observations. Thus, an HMM model results from the association of two stochastic processes: an unobservable process $Q(t)$ (states process) and an observable process $O(t)$.In general, the $Q(t)$ is a Markov chain which is supposed to be at each instant $t$ in a state $q_{t}=i(1 \leq i \leq K)$ which emits an observation $o_{t}$ with probability distribution $b_{i}(o)=P(o \backslash q=i)[30,31]$.

Let each region be represented by a sequence of feature vectors or observations sequence $O(t)$, defined as:

$$
O=o_{1}, o_{2}, \ldots, o_{T}
$$

Where $o_{t}$ is the features vector observed at time $\mathrm{t}$ and let the states sequence $Q$ given by:

$$
Q=q_{1}, q_{2}, \ldots, q_{T}
$$

HMM model can be considered as a probabilistic machine of finite states described by a set of nodes (or states) related together by arcs of transitions (see Fig.2). Each state $i(1 \leq i \leq K)$ at time instant $\mathrm{t}$ is associated with a probability distribution $b_{i}(o)=P\left(o \backslash q_{t}=i\right)$ of observation $o$ and with each transition from state $i$ to state $j$ is associated a transition probability $a_{i j}=P\left(q_{t}=j \backslash q_{t-1}=i\right)[30,31]$. If the observations are defined in a continuous space, then the probability density function of observation emission in state $j$ can be represented by a Gaussian mixture model(GMM) of M components, given by [26]:

$b_{j}\left(o_{t}\right)=\sum_{i=1}^{M} \frac{c_{i}}{\sqrt{(2 \pi)^{d}\left|\sum_{i}\right|}} \exp \left(-\frac{1}{2}\left(o_{t}-\mu_{i}\right)^{*} \cdot \sum_{i}^{-1} \cdot\left(o_{t}-\mu_{i}\right)\right)$

Where: $\mu_{i}$ and $\sum_{i}$ represent the mean and the covariance matrix of the $i^{t h}$ Gaussian component, $c_{i}$ is the weight of the $i^{t h}$ Gaussian satisfying the condition: $\sum_{i=1}^{M} c_{i}=1$

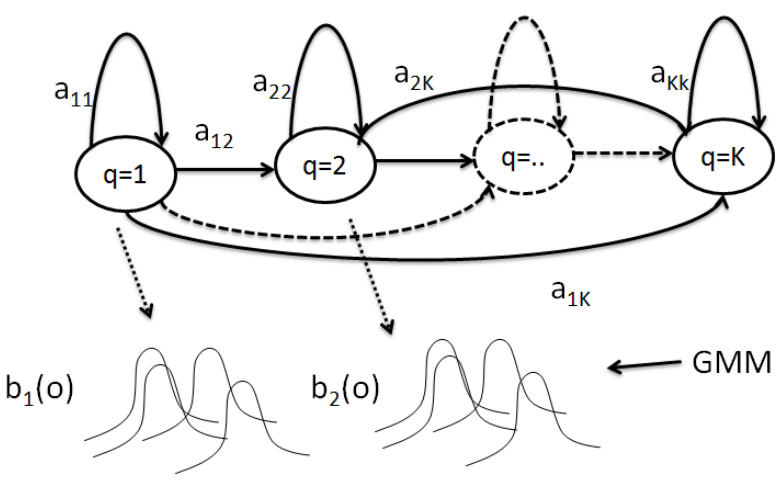

Fig. 2: Example of left- Right HMM Model [31].

The basic parameters of an HMM model are given as follows:

- The states transition matrix $A=\left\{a_{i j}\right\}$.

- The matrix of observation probability distribution $B=$ $\left\{b_{i}(o)\right\}$.

- The initial state probability $\pi=\left\{\pi_{i} \backslash=P\left(q_{1}=i\right)\right\}$ 
HMM can be written as $\lambda=(A, B, \pi)$

Given several HMM models $\lambda$ and given an input observations sequence $O$, the training phase consist to estimate the appropriate parameters of the HMM model that maximize the likelihood $P(O \backslash \lambda)$. Practically, the re-estimation of the HMM model parameters can be carried out using the Baum-Welch algorithm. The recognition phase has the objective to find the best HMM model that maximizes the probability $P(\lambda \backslash O)$. This phase can be carried out using the Viterbi algorithm.

2-HMM models based segmentation system : Fig.3 illustrates the diagram of our segmentation system. In the training phase, each region class is modelled by left-right HMM model of $N_{\text {states }}$, each one being represented by GMM model of $N_{G M M}$ Gaussian with diagonal covariance matrices. The parameters of HMM and GMM models are estimated using the HEREST command of the HTK tool, applied on the sequence of feature vectors extracted from the EMG signals of the training database. Furthermore, this estimation requires the reference text transcription that contents the class's sequence of each signal. The sequences of feature vectors are extracted using wavelet analysis applied on each EMG signal. This section will be described in the following section.

In the segmentation phase, the HVITE command of HTK tool uses the trained HMM models and constraints model (language model) for transcribing each input features vectors sequence in sequence of classes (AC and NAC) and detecting the boundaries of their segments $[26,32]$. The constraints problem consists to accept only the classes's sequence in which each AC label (class) is followed by NAC label is followed by NAC label (see Fig.1).

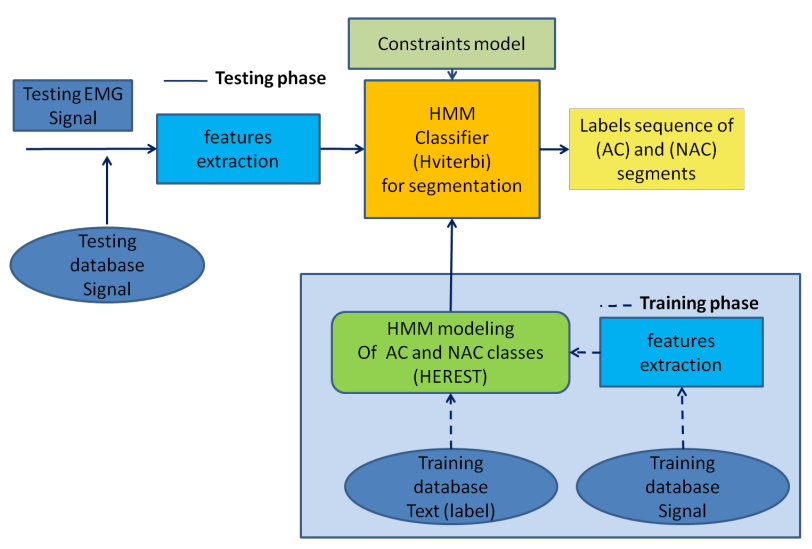

Fig. 3: HMM-models-based EMG signal segmentation.

Finally, the performance evaluation can be done using the HRESULTS command of HTK tool which compares each testing transcription of an EMG signal with its corresponding reference transcription [26]. The result of the segments identification is evaluated using the accuracy Acc defined in subsection A..

\section{Discrete wavelet transform based feature extraction}

Wavelet transform has largely been used as signal analysis technique in pattern recognition systems in order to extract a set of features from each analysis window (frame) of the signal of interest. The success of this technique essentially relies on the flexibility provided by the mother wavelet which choice depends on the properties of the data as well as on the targeted application.
For example, the orthogonality property between members of a wavelet family is desirable for compression purpose whereas the compactness property is desirable for the analysis and detection of impulsive data. Moreover, wavelet transform is particularly suitable for the analysis of data stemming from the real world because of its scale property that is compliant with many physical behaviors: long time events should be analyzed with slow long waveforms whereas short time or impulsive events should be analyzed with rapid short waveforms. The tool is thus suitable for correctly extracting the impulsive information of motor unit action potentials (MUAP) generated by EMG activation. Impulsive information can therefore be precisely localized and the information content precisely analyzed. To that aim, the authors of [10] proposed to use the first-order Hermite-Rodriguez function. The continuous wavelet transform has been used in [33] for the identification of the single action potentials in the time scale domain. The authors argue that the filtering effect of the volume conductor occurring between source signal taking its deep origin at the fiber level and MUAP detected signal at the skin surface produces different MUAP's that can be considered as dilated and attenuated versions of one single shape. This observation motivates the use of wavelet provided a mother wavelet with similar shape exists or can be created.

In the discrete form of the wavelet transform (DWT), decomposition coefficients are computed at different scales up to the maximum dyadic decomposition depth $p$ (to be a priori chosen or maximally fixed as $\log _{2}(N)$ where $N$ is the number of data samples in the analysis window). At each scale or decomposition level $j$, a set $d_{j}[n]$ (indexed by $n$ ) $N_{j}=\frac{N}{2^{j}}$ of wavelet coefficients is computed (the name $d$ is for detail coefficients). The last decomposition level $p$ produces the set $a_{p}[n]$ (the name $a$ is for approximation coefficients). Therefore, a discrete wavelet energy (DWE) can be computed at each scale as:

$$
\left\{\begin{array}{l}
E\left(d_{j}\right)=\sum_{n=0}^{N_{j}-1}\left|d_{j}[n]\right|^{2} \text { for } j=1, \ldots, p \\
E\left(a_{p}\right)=\sum_{n=0}^{N_{p}-1}\left|a_{p}[n]\right|^{2}
\end{array}\right.
$$

Finally, the set of features extracted from each analysis window of a signal is composed of wavelet coefficient energies $E\left(d_{1}\right), E\left(d_{2}\right), \ldots, E\left(d_{p}\right), E\left(a_{p}\right)$, which makes an energy analysis on consecutive spectral bands of the data (from the highest to the lowest when $j$ goes from 1 to $p$ ). In order to extract other possible descriptors, the authors in [27] have used the DWE normalized on total energy of window analysis, the logarithm of wavelet energy (LWE) and the Wavelet Cepstral Coefficient (WCC) computed from the discrete cosine transform (DCT) of LWE (see Fig.4). In this work, we investigated the impact of these descriptors on the performance results of EMG signal segmentation. Hence different experiences have been carried out to search for the optimal configuration.

\section{Data base description}

The HMM based method is applied to real sEMG signals. It has been recorded on different muscles which characterize the gait. These signals have several EMG activity bursts and are recorded in ecological conditions within the French National 


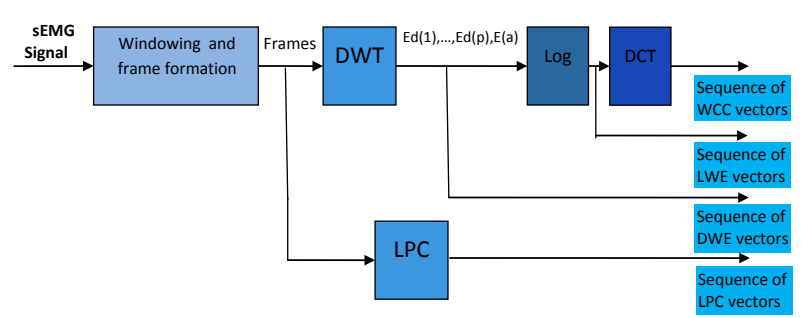

Fig. 4: Process for the extraction of LPC / DWE / LWE / WCC features [27].

Project ECOTECH [18]. The group was constituted of nine healthy subjects and eight Parkinsonian subjects recorded during the ECOTECH project. This study was approved by the local ethics committee and subjects provided written consent prior to commencement.

We have tested the proposed method for surface EMG signals recorded during the ECOTECH experiment. Patients were prepared for electrodes placement by shaving the skin and cleaning it with alcohol wipes. Differential EMG sensors were placed on the muscle belly parallel to the main direction of muscle fibres in accordance with study on the innervation zone [34]. Data were collected using an on board system of 16 wearable sensors (bandwidth 20-450 Hz, 16 bits per sample, $1926 \mathrm{~Hz}$ sampling rate, Delsys Trigno, Natick, USA) with SNR = 3.31 dB. Each sensor recorded the activity of a single muscle. In this work, we selected the data collected from the right soleus muscle ( $R S o l)$ which is predominantly involved in gait and shows high energy. For this muscle, all EMG bursts were previously manually segmented by an expert. The data base description is reported in tables I and II.

The group of eight Parkinson's patients had a median age of 59.5 years [54-65.7, first and third quartiles] and was composed of two women and six men with a moderate bilateral deficit and a median UPDRS score of 7.5 [3.75-13.25], score obtained under anti-parkinsonian medication (ON state). The control group of nine healthy people had a median age of 38 years [38-49] and consisted of one woman and eight men. No motor disability or dysfunction were a prerequisite to be part of this latter. The electromyographic activities were collected during continuous walking sequences for which the conditions were the same between groups: free (natural gait), rectilinear and without obstacles or obstructions. More specifically for the patients, the gait tests were carried out in ON state.

\section{RESUlTS AND DISCUSSION}

The proposed HMM based method was applied to segment the real sEMG signals automatically. The following section describes the performance tools used for an objective comparison of the segmentation results. Then, an experimental setup is carried out in order to get the optimal configuration of the segmentation system. Statistical segmentation results are also provided and the obtained results are compared with those of the state-of-the-art methods reported in [15].

This expertise was conducted within the ECOTECH project [18] where the EMG signal segmentation has been achieved by biomedical researchers using visual inspection.

\section{A. Performance evaluation tools}

In order to evaluate the performance of the segmentation task, we have considered two main criteria, namely the classification accuracy $(A c c)$ and the error rate $(R e)$. The $A c c$ is defined in Eq.(6) and is used to evaluate the number of correctly detected EMG burst activities using the HRESULTS command of HTK tool [26].

$$
A c c=\frac{N-D-S-I}{N}
$$

$N$ represents the total number of segments labels in the reference transcriptions of EMG signals, $D$ is the number of deleted labels, $S$ is the number of substituted labels and $I$ is the number of inserted labels.

The $R e$ performance criterion is used for the fine detection of the beginning (onset) and the end (offset) of each EMG burst activity. In order to evaluate $R e(\%)$, we have used a binary representation of our sEMG signal according to:

$B[n]=\left\{\begin{array}{rr}0 & \text { if the decision made for the } n \text {-th sample is 'no activity' } \\ 1 & \text { if the decision made for the } n \text {-th sample is 'activity' }\end{array}\right.$

$$
R e=\operatorname{Prob}\left(B[n] \neq B_{e x}[n]\right)
$$

where $B_{e x}[n]$ refers to the exact value of our binary signal representation given by the expert and Prob refers to the probability of a given event.

In this work, we have also achieved the performance's analysis by computing the mean and standard deviation (STD) values of the burst duration values as well as the corresponding absolute latency error (ALE) between the mean of the experimental duration and the mean of the expert duration as given in Eq.(8).

$A L E=\mid$ Mean of real bursts durations - Mean of estimated bursts durations $\mid$

\section{B. Optimal configuration of the segmentation system}

The purpose of this section is to describe the experimental setup that will give the best configuration of our system by evaluating the proposed HMM method's performance for two types of signals, namely real EMG signals of healthy subjects and real EMG signals of Parkinsonian subjects.

We present in this section a number of experiments we carried out to find the optimal configuration of our framework that gives the best performance To find the optimal parameters of the HMM (state number and gaussian components number).

Three experiments are processed in order:

(1) to compare the performance of the DWE, LWE and WCC descriptors to other features commonly used in the literature such as prediction analysis (LPC: Linear Prediction Coefficients); 


\begin{tabular}{|c|c|c|c|c|c|c|}
\hline \multicolumn{2}{|c|}{ Subjects } & Number of EMG bursts & Mean of real EMG burst duration (ms) & STD of real EMG burst duration (ms) & Signal length (samples) & Signal duration $(\mathrm{s})$ \\
\hline \multirow{5}{*}{ Training phase } & Control $_{1}$ & 22 & 1037.7000 & 98.5539 & 50206 & 26.0685 \\
\hline & Control $_{2}$ & 10 & 1059.7000 & 288.3023 & 21595 & 11.2128 \\
\hline & Control $_{3}$ & 11 & 1068.1000 & 85.4207 & 27733 & 14.3998 \\
\hline & Control $_{4}$ & 11 & 1069.3000 & 103.7267 & 28396 & 14.7441 \\
\hline & Control $_{5}$ & 11 & 809.9091 & 33.3090 & 21500 & 11.1635 \\
\hline \multirow{4}{*}{ Testing phase } & Control $_{6}$ & 6 & 1031.7000 & 73.5193 & 14853 & 7.7121 \\
\hline & Control $_{7}$ & 6 & 1013.3000 & 321.9687 & 12576 & 6.5298 \\
\hline & Control $_{8}$ & 12 & 898.0833 & 48.5264 & 27629 & 14.3458 \\
\hline & Control $_{9}$ & 26 & 969.2308 & 104.8490 & 55024 & 28.5702 \\
\hline
\end{tabular}

Table. I

DESCRIPTION OF SEMG SIGNALS FOR HEALTHY SUBJECTS.

\begin{tabular}{|c|c|c|c|c|c|c|}
\hline \multicolumn{2}{|c|}{ Subjects } & Number of EMG bursts & Mean of real EMG burst duration (ms) & STD of real EMG burst duration (ms) & Signal length (samples) & Signal duration $(\mathrm{s})$ \\
\hline \multirow{4}{*}{ Training phase } & Park $_{1}$ & 10 & 1225.3000 & 65.0898 & 55024 & 28.5702 \\
\hline & Park $_{2}$ & 10 & 959.2000 & 121.2910 & 17587 & 9.1317 \\
\hline & Park $_{3}$ & 5 & 1362 & 233.4309 & 9371 & 4.8657 \\
\hline & Park $_{4}$ & 37 & 933.8108 & 128.3433 & 76296 & 39.6152 \\
\hline \multirow{4}{*}{ Testing phase } & Park $_{5}$ & 10 & 766.2000 & 53.1012 & 21739 & 11.2876 \\
\hline & Park $_{6}$ & 9 & 990.5656 & 55.1750 & 17170 & 8.9152 \\
\hline & Park $_{7}$ & 5 & 773.8000 & 157.9421 & 8617 & 4.4742 \\
\hline & Park $_{8}$ & 5 & 652.2000 & 80.6021 & 8953 & 4.6487 \\
\hline
\end{tabular}

Table. II

DESCRIPTION OF SEMG SIGNALS FOR PARKINSONIAN SUBJECTS.

(2) to find the optimal analyzing frame duration;

(3) to search for the optimal combination of decomposition level and mother wavelet;

(4) to analyze the performance results of our framework and to compare them with the results obtained using other state-of-the-art existing methods.

In order to deeply evaluate the performance of our method, we carried out the first experiment for different numbers of HMM states $N_{\text {states }}$, different numbers of Gaussians for GMM modelling $N_{G M M}$ and different levels of wavelet decomposition $L_{\text {decomp }}$ or $P$ order for LPC descriptor. The mother wavelet 'Sym4' was chosen with $66.45 \mathrm{~ms}$ window durations as recommended in [35] for EMG pattern recognition. The best descriptors with the optimal parameters were chosen for the second experiment of window duration study then for the optimal wavelet configuration. Finally a comparison with state of the art methods is carried out.

1-Performance comparison of the descriptors: This section shows evaluation results of the DWE descriptor for the task of surface EMG signal segmentation. The performance analysis is compared to LPC descriptor, LWE (Log Wavelet decomposition based Energy) descriptor and WCC descriptor. In this experiment, we search for the optimal parameters configuration that gives the best $A c c$ and $R e$. For each descriptor, we have varied the state number for each experiment $N_{\text {states }}=(2,3,4,5,6,7,8,9,10)$, the number of components in GMM modelling $N_{G M M}=(1,2,3,6,12,24,48)$ and the order $P=(2,3,4,5,6,7,8,9,10)$ of LPC descriptor. The analysis frame duration is fixed to $66.45 \mathrm{~ms}$ (which represents 128 samples) and the mother wavelet is chosen to 'Sym4' and the wavelet decomposition level $L_{\text {decomp }}=4$. The obtained results are given in Table III. The latter shows the optimal configurations in terms of number of Gaussians $N_{G M M}$ and number of states $N_{\text {states }}$ for each wavelet descriptor DWE, LWE and WCC as well as the optimal order $P$ added for the LPC descriptor. The
$A c c$ and $R e$ values are given for these optimal configurations. In order to evaluate the robustness of each descriptor regarding the variations of $N_{G M M}$ and $N_{\text {states }}$, the mean and STD values of $R e$ are reported by computing the $R e$ values when considering all the values of the tested parameter sets. From all these results, we adopted the following rules in order to identify the best configuration of the system. First, we examine the best accuracy results. Second, when multiple best configurations exist, the best Re values are considered also taking into account the robustness results. Results shown in Table III highlight the performance gain of the wavelet descriptors that all show $100 \%$ of Acc compared to the LPC descriptor showing the bad score of $87.34 \%$. Now, the best descriptor is WCC with $4.88 \%$ for the $R e$ value. However, the WCC descriptor requires more calculus and is more influenced by the variations of the state number and the gaussian number (mean of 7.41 and standard deviation of 0.51). The next candidate with a weak $R e$ value is LWE also showing better $R e$ statistics than WCC (in particular with an STD value which is about 4 times lower). Hence, in the following sections, we choose the LWE descriptor by taking a 2 -states number and a 3-gaussians number.

2-Influence of the window duration: After choosing the feature's descriptor, we investigate the appropriate analysis frame duration. This section aims at investigating performance improvements by taking into account the advantages of wavelet analysis appropriate for the non stationary case of EMG signals. We have varied the analysis frame duration with 'Sym4' wavelet, number of GMM $N_{G M M}=3$, number of states $N_{\text {states }}=2$ and decomposition level equal to $L_{\text {decomp }}=4$ for LWE (Log Wavelet decomposition based Energy) descriptor. Table IV shows the $A c c$ and $R e$ values for different values of analysis frame duration. The best performance is obtained for analysis frame duration equal to $66.45 \mathrm{~ms}$ which gives $A c c$ and $R e$ values equal to $100 \%$ and the lowest $R e$ equal to $5.37 \%$.

The following subsection focuses on the performance analysis for the optimal choice of mother wavelet. 


\begin{tabular}{|c|c|c|c|c|}
\hline Descriptors & $L P C^{*}$ & $D W E$ & $L W E$ & $W C C$ \\
\hline Optimal parameters & $N_{G M M}=24$ & $N_{G M M}=3$ & $N_{G M M}=3$ & $N_{G M M}=48$ \\
& $N_{\text {states }}=2$ and $P=2$ & $N_{\text {states }}=3$ & $N_{\text {states }}=2$ & $N_{\text {states }}=2$ \\
\hline$A c c \%$ & 87.34 & 100 & 100 & 100 \\
\hline$R e \%$ & 43.48 & 6.16 & 5.37 & 4.88 \\
\hline Mean of $R e \%$ & 49.14 & 6.24 & 6.23 & 7.41 \\
\hline STD of $R e \%$ & 0.731 & 0.083 & 0.138 & 0.510 \\
\hline
\end{tabular}

Table. III

PerformanCE COMPARISON OF THE $A c c \%$ FOR DWE, LPC, LWE AND WCC FEATURES USING 'Sym4' AND $L_{d e c o m p}=4$ WITH ANALYSIS FRAME DURATION EQUAL TO $66.45 \mathrm{~ms}$ FOR THE HMM OPTIMAL PARAMETERS.THE MEAN AND STD VALUES ARE STATISTICAL RESULTS EVALUTED BY CONSIDERING $N_{G M M}$ AND $N_{\text {states }}$ RANGES PLUS $P$ RANGE FOR LPC DESCRIPTOR. THE STAR * INDICATES THAT Re WAS CALCULATED ONLY TAKING INTO ACCOUNT CORRECTLY CLASSIFIED SEQUENCES

\begin{tabular}{|c|c|c|c|c|c|c|c|c|}
\hline Analysis frame duration $(m s)$ & 16.61 & 33.22 & 49.84 & 66.45 & 83.07 & 99.68 & 116.30 & 132.91 \\
\hline \hline$A c c \%$ & 80.38 & 91.14 & 97.47 & 100 & 100 & 99.37 & 100 & 100 \\
\hline$R e \%$ & 6.41 & 5.39 & 5.33 & 5.37 & 5.505 & 5.61 & 5.81 & 8.46 \\
\hline
\end{tabular}

Table. IV

Performance COMParison of the $A c c(\%)$ And $R e(\%)$ FOR DWE FEATURES Using 'Sym4' For DifFERENT ANALYSIS FRAME DURATIONS FOR THE HMM OPTIMAL PARAMETERS.

3- Choice of the mother wavelet: Many studies of surface EMG analysis have concluded that the Daubechies $(\mathrm{Db})$ wavelet family is the most suitable wavelet for sEMG signal analysis [36-38]. In [35], the authors have concluded that the 'Sym4' is the most appropriate one for EMG pattern recognition. This experiment aims at selecting the optimal mother wavelet order within its family for an analysis window durations equal to $66.45 \mathrm{~ms}$, a number of GMM $N_{G M M}=3$, a number of states $N_{\text {states }}=2$ with a decomposition level varying between 1 to $\log 2$ of samples number of analysis window (max level=7). In this work, we consider the following wavelet families:

- the Daubechies family with orders 1 to $8: \mathrm{Db} 1, \mathrm{Db} 2, \ldots$, Db8;

- the Symlets family with orders 1 to $8: \operatorname{Sym} 1, \operatorname{Sym} 2, \ldots$, Sym8;

- the Coiflets family with orders 1 to 5: Coif1, Coif2,..., Coif5.

The Acc, $R e$ and $L_{\text {decomp }}$ values are reported in Tables V, VI and VII for each of the three wavelet families, respectively. For all the families, the optimal decomposition level decreases with the augmentation of the mother wavelet order. The results highlight the performance robustness of the proposed approach in terms of $A c c$ value where we notice that the mean values of $A c c$ are greater than $97.47 \%$ and the mean of error rate $R e$ are lower than $5.70 \%$. In particular, we also notice that the 'Coif5' wavelet for decomposition level equal to $L_{\text {decomp }}=4$ leads to the minimum of $R e=4.68 \%$ and the maximum of $A c c=100 \%$. This latter result shows improved performance in terms of error rate compared to WCC performance as reported in Table III. The improvement of the results is consistent with the step by step experimental strategy we follow, which purpose is to propose a tuned set for the configuration of the system, without requiring too much exhaustive empirical calculus. We thus retain this last result as a new starting point for further comparison studies that will be presented in section $\mathrm{C}$.

\section{Statistics of the bursts duration estimation}

In this part of our work, we conduct a performance analysis of the segmentation task using statistics on the segmented bursts duration values (mean, STD and ALE). The results are obtained with an analysis frame duration equal to $66.45 \mathrm{~ms}$, a number of GMM $N_{G M M}=3$, a number of states $N_{\text {states }}=2$ and a decomposition level equal to $L_{\text {decomp }}=4$ with the mother wavelet 'Coif 5'.

Table VIII presents the mean and the STD of the estimated bursts duration values, the estimated number of EMG activity bursts and ALE for the healthy's and Parkinson's subjects from the sEMG records of right soleus muscle. The table shows that the estimated numbers of bursts are compliant with the expert numbers. The results also show low ALE values (less than 46 ms for all subjects) except for one Parkinson's subject (showing $80 \mathrm{~ms})$.

Fig.5 and Fig.6 show illustrative examples of a real sEMG signal for right Soleus muscle of control and Parkinson's subjects respectively. The signal is segmented in AC and NAC parts using HMM method for $N_{G M M}=3, N_{\text {states }}=2, L_{\text {decomp }}=4$, wavelet $=$ Coif 5 and frame length $=66.45 \mathrm{~ms}$ for an error rate equal to $R e=2.71 \%$ for control subject and for Parkinson's subject $R e=6.06 \%$.

\section{Performance comparison with some state-of-the-art segmen- tation methods}

In order to compare our method with state-of-the-art segmentation methods, we have considered the five methods presented 


\begin{tabular}{|c|c|c|c|c|c|c|c|c|c|c|c|}
\hline \multicolumn{2}{|c|}{ Daubechies } & $D b 1$ & $D b 2$ & $D b 3$ & $D b 4$ & Db5 & Db6 & Db7 & Db8 & Mean & STD \\
\hline \hline \multirow{3}{*}{$66.45 \mathrm{~ms} s$} & $L_{\text {decomp }}$ & 7 & 5 & 5 & 5 & 4 & 4 & 4 & 4 & $/ /$ & $/ /$ \\
\cline { 2 - 12 } & $A c c \%$ & 97.47 & 100 & 100 & 100 & 100 & 100 & 100 & 100 & $/ /$ & $/ /$ \\
\cline { 2 - 12 } & $R e \%$ & 5.20 & 5.08 & 5.28 & 5.47 & 5.53 & 4.98 & 5.23 & 5.02 & 5.22 & 0.199 \\
\hline
\end{tabular}

Table. $\mathrm{V}$

Performance ReSUlts in terms of $A c c(\%), R e(\%)$ AND OPTIMAl $L_{\text {decomp }}$ FOR LWE fEATURES USING DAUBECHIES WAVELET FAMILY.

\begin{tabular}{|c|c|c|c|c|c|c|c|c|c|c|c|}
\hline \multicolumn{2}{|c|}{ Symlets } & Sym1 & Sym2 & Sym3 & Sym4 & Sym5 & Sym6 & Sym7 & Sym8 & Mean & STD \\
\hline \hline \multirow{3}{*}{$66.45 \mathrm{~ms} s$} & $L_{\text {decomp }}$ & 7 & 5 & 5 & 5 & 4 & 4 & 4 & 4 & $/ /$ & $/ /$ \\
\cline { 2 - 13 } & Acc \% & 100 & 100 & 100 & 100 & 100 & 100 & 100 & 100 & /I & /I \\
\cline { 2 - 12 } & $R e \%$ & 5.20 & 5.08 & 5.28 & 5.21 & 5.18 & 5.70 & 5.20 & 5.30 & 5.268 & 0.186 \\
\hline
\end{tabular}

Table. VI

Performance ReSUlts in terms of $A c c(\%), R e(\%)$ AND optimal $L_{\text {decomp }}$ FOr LWE FEATURES USING SYMLETS WAVELET FAMILY.

\begin{tabular}{|c|c|c|c|c|c|c|c|c|}
\hline \multicolumn{2}{|c|}{ Coiflets } & Coif1 & Coif2 & Coif3 & Coif4 & Coif5 & Mean & STD \\
\hline \hline \multirow{3}{*}{$66.45 m s$} & $L_{\text {decomp }}$ & 6 & 4 & 4 & 4 & 4 & $/ /$ & $/ /$ \\
\cline { 2 - 9 } & $A c c \%$ & 100 & 100 & 100 & 100 & 100 & $/ /$ & $/ /$ \\
\cline { 2 - 9 } & $R e \%$ & 4.91 & 4.99 & 5.26 & 5.23 & 4.68 & 5.014 & 0.2399 \\
\hline
\end{tabular}

Table. VII

PeRformance Results in terms of $A c c(\%), R e(\%)$ AND OPTIMAl $L_{\text {decomp }}$ FOR LWE FEATURES USING COIFLETS WAVELET FAMILY.

\begin{tabular}{|c|c|c|c|c|c|c|c|c|c|}
\hline \multicolumn{2}{|c|}{ Subject } & Control $_{6}$ & Control $_{7}$ & Control $_{8}$ & Control $_{9}$ & Park $_{5}$ & Park $_{6}$ & Park $_{7}$ & Park $_{8}$ \\
\hline \hline \multirow{3}{*}{ Real burst duration } & Mean(ms) & 1031.7000 & 1013.3000 & 898.0833 & 969.2308 & 766.2000 & 990.5556 & 773.8000 & 652.2000 \\
\cline { 2 - 11 } & STD (ms) & 73.5192 & 321.9687 & 48.5264 & 104.8490 & 53.1012 & 55.1750 & 157.9421 & 80.6021 \\
\cline { 2 - 11 } & Number & 6 & 6 & 12 & 26 & 10 & 9 & 5 & 5 \\
\hline \multirow{3}{*}{ Estimated burst duration } & Mean (ms) & 1003.7020 & 1044.0991 & 925.2054 & 1003.3472 & 719.77 & 959.2504 & 693.7117 & 616.0029 \\
\cline { 2 - 10 } & STD (ms) & 57.2459 & 350.6888 & 48.3647 & 121.5427 & 125.0333 & 49.9449 & 124.7589 & 72.9712 \\
\cline { 2 - 10 } & Number & 6 & 6 & 12 & 26 & 10 & 9 & 5 & 5 \\
\hline Performance & ALE (ms) & 27.95 & 31.09 & 27.12 & 34.11 & 46.43 & 31.30 & 80.0883 & 36.1971 \\
\hline
\end{tabular}

Table. VIII

MEAN, STANDARD DEVIATION (STD) AND ABSOLUTE LATENCY ERROR (ALE) OF EMG BURST ACTIVITY DURATION FOR RSOL RIGHT soleus MUSCLE FOR HEALTHY AND PARKINSON'S SUBJECTS.

in [15]. Table IX presents $R e$ values for the healthy and Parkinson's subjects of sEMG records from the right Soleus muscle. The results highlight the performance gain of the proposed HMM approach compared to the other existing methods. In order to facilitate the reading of the results, the Mean and STD values are graphically displayed in Fig.7. In order to highlight the differences between the two classes, the graphical statistical results are also presented in Fig. 8 for both categories. From these graphs, we notice that, the HMM method seems to be the most appropriate for an automatic EMG activity monitoring for all the subjects (Fig.7). More specifically, by comparison with the other state-of-the-art methods, the segmentation is far better for the control subjects and also shows a slight improvement for the Parkinsonian's subjects (Fig.8).
Table X presents the comparative ALE values and estimated numbers of EMG activity bursts for the Control $_{6}$ and Park $_{5}$ subjects of sEMG records from the right Soleus muscle. The results highlight the performance gain of the proposed HMM approach compared to the other existing methods, in particular w.r.t. the number of EMG activity bursts. For the Parkinson case, all methods except our failed in estimating the right number of bursts. The ALE values for our method are also always the lowest among the concurrent methods.

\section{E. Recommendation and discussion}

Several techniques already exist for EMG signal activity segmentation, but the detection performance is far from being satisfactory in difficult cases like in neurodegenerative EMG activity. This work represents a further step towards developing efficient 


\begin{tabular}{|c|c|c|c|c|c|c|c|}
\hline Subject & DoubleTh & Wavelet & TKEO & RMS & SampEn & FM-ALED & HMM \\
\hline \hline Park $_{5}$ & 20.11 & 22.04 & 18.96 & 18.09 & 17.65 & 9.78 & 6.06 \\
Park $_{6}$ & 22.00 & 20.63 & 22.54 & 12.90 & 14.54 & 5.83 & 3.16 \\
Park $_{7}$ & 19.82 & 18.65 & 15.27 & 9.40 & 14.50 & 7.92 & 10.35 \\
Park $_{8}$ & 17.08 & 21.94 & 17.59 & 16.06 & 13.72 & 10.77 & 5.55 \\
Control $_{6}$ & 22.96 & 14.42 & 10.81 & 8.03 & 7.53 & 4.38 & 2.71 \\
Control $_{7}$ & 18.04 & 17.77 & 12.27 & 11.14 & 8.83 & 5.07 & 3.07 \\
Control $_{8}$ & 19.74 & 20.43 & 15.76 & 15.27 & 23.46 & 7.62 & 3.02 \\
Control $_{9}$ & 17.08 & 22.75 & 15.47 & 18.92 & 9.55 & 7.60 & 3.52 \\
\hline
\end{tabular}

Table. IX

COMPARISON OF ERROR RATE Re WITH THEIR MEAN AND STANDARD DEVIATION VALUES OF EMG ACTIVITY BURST DETECTION FOR RSOL RIGHT soleus FOR HEALTHY AND PARKINSON's SUBJECTS.

\begin{tabular}{|c|c|c|c|c|c|c|c|c|c|}
\hline \multicolumn{2}{|l|}{ Subject } & Real burst duration & DoubleTh & Wavelet & TKEO & RMS & SampEn & FM-ALED & HMM \\
\hline \multirow{3}{*}{ Estimated burst duration for $\mathrm{Control}_{6}$} & Mean (ms) & 1031.70 & 209.47 & 604.67 & 549.00 & 904.29 & 891.00 & 986.47 & 1003.70 \\
\hline & STD (ms) & 73.52 & 157.85 & 434.41 & 604.15 & 394.28 & 520.51 & 128.60 & 57.24 \\
\hline & Number & 6 & 19 & 9 & 14 & 7 & 8 & 6 & 6 \\
\hline Performance for Control $_{6}$ & ALE (ms) & $/ /$ & 822.22 & 427.03 & 482.70 & 127.41 & 140.07 & 45.18 & 27.95 \\
\hline \multirow{3}{*}{ Estimated burst duration for $\operatorname{Park}_{5}$} & Mean (ms) & 766.20 & 281.18 & 327.46 & 284.20 & 300.88 & 327.55 & 667.56 & 719.77 \\
\hline & STD (ms) & 53.10 & 201.96 & 180.38 & 177.59 & 281.33 & 105.15 & 16.33 & 125.03 \\
\hline & Number & 10 & 17 & 13 & 15 & 34 & 11 & 9 & 10 \\
\hline Performance for Park $k_{5}$ & ALE (ms) & $1 /$ & 485.02 & 438.73 & 482.00 & 465.31 & 438.65 & 98.64 & 46.43 \\
\hline
\end{tabular}

Table. X

MEAN, STANDARD DEVIATION (STD) AND ABSOLUTE LATENCY ERROR (ALE) OF EMG BURST ACTIVITY DURATION FOR

RSOL RIGHT soleus MUSCLE OF Control 6 AND Park 5 SUBJECTS USING DIFFERENT SEGMENTATION METHODS.

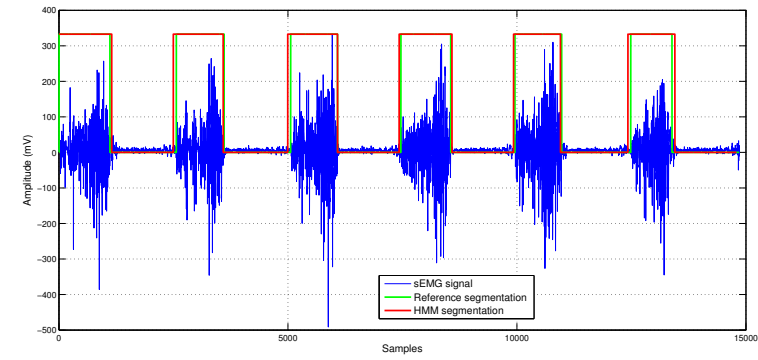

Fig. 5: Automatic segmentation of real sEMG signal of the control subject $\left(\right.$ Control $_{6}$ ) with HMM method for right Soleus muscle with $R e=2.71 \%\left(N_{G M M}=3, N_{\text {states }}=2, L_{\text {decomp }}=\right.$ 4, wavelet $=$ Coif 5 and frame length $=66.45 \mathrm{~ms}$.)

automatic segmentation solution which is an important task in raw data processing in order to be able to exploit massive EMG datasets necessary for the development of systems that can be used as genuine medical diagnosis supports in future, especially in Parkinson's disease diagnostic. The proposed approach can be seen as an improvement of the EMG activity detection problem in particular because the study has been carried out on the ECOTECH dataset gathering ecological difficult situations close to conditions that can be encountered in the targeted applications. In this context, our proposed HMM method has been applied to real EMG signals recorded for healthy and Parkinson's subjects. Different experiments have been carried out to find the optimal configuration of the segmentation system that gives the best performance in terms of accuracy and error rate. The results have

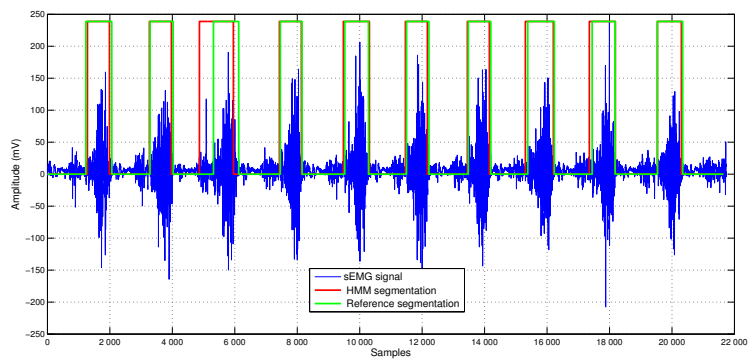

Fig. 6: Automatic segmentation of real sEMG signal of the Parkinson's subject $\left(\right.$ Park $_{5}$ ) with HMM method for right Soleus muscle with $R e=6.06 \%\left(N_{G M M}=3, N_{\text {states }}=2, L_{\text {decomp }}=\right.$ 4 , wavelet $=$ Coif 5 and frame length $=66.45 \mathrm{~ms}$.)

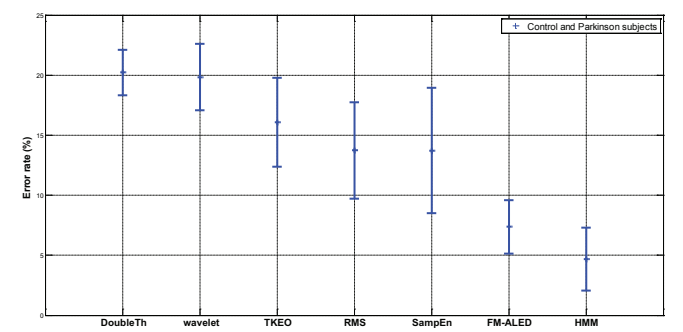

Fig. 7: Comparison of error rate Re showing mean and standard deviation of burst EMG activity detection for RSol right soleus for healthy and Parkinson's subjects. 


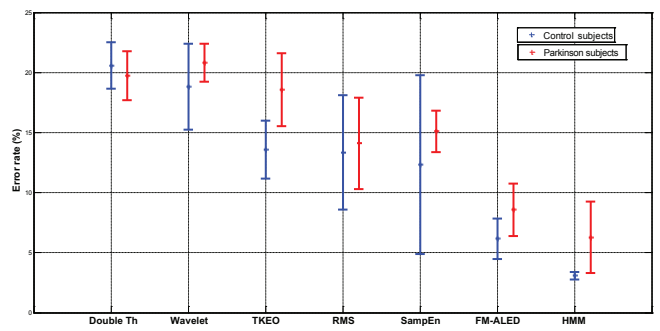

Fig. 8: Comparison of error rate Re showing mean and standard deviation of burst EMG activity detection for RSol right soleus for healthy and Parkinson's subjects.

shown clear improvements of the system performance gained step by step from consecutive experiments. We summarize and discuss here the main improvements made out of this study and highlight some perspectives for this research work.

- The DWT analysis presents the first improvements compared to the LPC analysis whatever the wavelet descriptor type (DWE, LWE and WCC). This analysis gives the accuracy of $100 \%$. Particularly, the LWE descriptor is shown to be more appropriate for the segmentation task. These results have been obtained by taking the best configuration of HMM models of 2 states associated each one with a GMM model of 3 gaussian components, analysis window of $66.45 \mathrm{~ms}$ duration and giving error rates around $5 \%$ whatever the family of mother functions.

It can be noticed that the optimal decomposition level decreases by increasing the order of the Daubechies wavelet family (this is also true for the other families). This can be explained by the fact that the length of the associated filter used for wavelet coefficients computation increases with the Daubechies order. At the first Daubechies orders, filter lengths are rather small which remains applicable for filtering purpose with a small number of data samples that are considered at the deepest decomposition levels. The order increasing, the filter length increasing thus requiring data with a higher number of samples for a correct filtering application. Therefore limiting the optimal decomposition level values to 4 seems to be a appropriate choice in this context. Experimental results show that the Coiflet wavelet function with level decomposition of 4 presents the best error rate of $4.68 \%$. Also the Sym2 and Db6 give a good performance too.

- The obtained segmentation performance in terms of error rate, absolute latency error and the number of burst EMG activity out performs those of the existing methods from the literature used in our comparative study (see Tables IX and X). A key point stemming from these results is the ability of the proposed method to estimate the correct number of bursts whatever the subjects under study. Having access to the number of bursts with their durations is essential for the monotoring of pakinsonian subjects and particularly in the frame of the following of a rehabilitation protocol. To that aim, we also recommend the use of supervised techniques even if labelling data can be controversial and difficult to obtain from experts. These methods are far less common than current state-of-the-art unsupervised methods.

- The proposed HMM technique is most appropriate for EMG signal segmentation of healthy subjects where we have observed a significant performance gain in terms of error rate (see Fig.8).

- In this study, we have used the discrete wavelet transform as feature descriptor because this decomposition is more adapted than the Fourier decomposition to extract the impulsive information of the action potentials of the motor units (provided that the mother wavelet is well selected).

- Our proposed HMM method is robust to the choice of the mother wavelet type since we have concluded that all the tested mother wavelets family gave an error rate close to 5 $\%$ and classification accuracy close to $98 \%$.

\section{CONCLUSION}

Muscle activity detection of human skeletal muscle has important clinical applications. In this paper, we have proposed a novel framework for muscle activity detection based on the EMG signal segmentation using Hidden Markov models combined with DWT analysis. Different descriptors extracted from this analysis have been used and the results have shown that the HMM models combined with LWE descriptor extracted using the Coiflet 5 with decomposition level 4 give the best performance in terms of accuracy and error rate. A comparative study with state-of-the-art methods confirms the effectiveness of our approach. The proposed approach offers a good detection performance suitable for clinical applications. It may be a useful tool for analysing the EMG signals recorded during the assessment of postural adjustment of patients suffering from Parkinson's disease. As perspectives, we believe the use of an enlarged EMG dataset including all the EMG channels of ECOTECH distribution would be necessary to reinforce these first results and move a step towards the real-life application of the method. Another important issue (not treated in this work) would be to analyze the method's performance for EMG signals relative to other types of neurodegenerative diseases, e.g. Huntington and ALS (Amyotrophic lateral sclerosis).

\section{ACKNOWLEDGMENTS}

The present paper used collected data from the French national project ECOTECH supported by the French National Agency for research under the contract No. ANR-12-TECS-0020.

\section{REFERENCES}

[1] R Merletti, D Farina, M Gazzoni, A Merlo, et al. Surface electromyography: A window on the muscle, a glimpse on the central nervous system. European Journal of Physical and Rehabilitation Medicine, 37(1):57, 2001.

[2] Seth L Pullman, Douglas S Goodin, Anthony I Marquinez, Samer Tabbal, and Michael Rubin. Clinical utility of surface EMG: report of the therapeutics and technology assessment subcommittee of the american academy of neurology. Neurology, 55(2):171177, 2000. https://doi.org/10.1212/wnl.55.2.171

[3] Paolo Bonato, Tommaso D'Alessio, and Marco Knaflitz. A statistical method for the measurement of muscle activation intervals from surface myoelectric signal during gait. IEEE 
Transactions on Biomedical Engineering, 45(3):287-299, 1998. https://doi.org/10.1109/10.661154

[4] Gerhard H Staude, Werner M Wolf, Ulrich Appel, and Reinhard Dengler. Methods for onset detection of voluntary motor responses in tremor patients. IEEE Transactions on Biomedical Engineering, 43(2):177-188, 1996. https://doi.org/10.1109/10.481987

[5] Silvestro Micera, Angelo M Sabatini, and Paolo Dario. An algorithm for detecting the onset of muscle contraction by EMG signal processing. Medical Eng. \& Physics, 20(3):211-215, 1998. https://doi.org/10.1016/S1350-4533(98)00017-4

[6] Lev Vaisman, José Zariffa, and Milos R Popovic. Application of singular spectrum-based change-point analysis to EMG-onset detection. Journal of Electromyography and Kinesiology, 20(4):750-760, https://doi.org/2010. 10.1016/j.jelekin.2010.02.010

[7] Qi Xu, Yazhi Quan, Lei Yang, and Jiping He. An adaptive algorithm for the determination of the onset and offset of muscle contraction by EMG signal processing. IEEE Transactions on Neural Systems and Rehabilitation Engineering, 21(1):65-73, 2012. https://doi.org/10.1109/TNSRE.2012.2226916

[8] Usman Rashid, Imran Khan Niazi, Nada Signal, Dario Farina, and Denise Taylor. Optimal automatic detection of muscle activation intervals. Journal of Electromyography and Kinesiology, 48:103111, 2019. https://doi.org/10.1016/j.jelekin.2019.06.010

[9] Jie Liu, Dongwen Ying, and William Zev Rymer. EMG burst presence probability: A joint time-frequency representation of muscle activity and its application to onset detection. Journal of Biomechanics, 48(6):1193-1197, 2015. https://doi.org/10.1016/j.jbiomech.2015.02.017

[10] Andrea Merlo, Dario Farina, and Roberto Merletti. A fast and reliable technique for muscle activity detection from surface EMG signals. IEEE Transactions on Biomedical Engineering, 50(3):316-323, 2003. https://doi.org/10.1109/TBME.2003.808829

[11] Hsiu-Jen Liu, Kuu-Young Young, et al. An adaptive upper-arm EMG-based robot control system. International Journal of Fuzzy Systems, 12(3):181-189, 2010. https://doi.org/10.30000/IJFS.201009.0001

[12] Xiaoyan Li, Ping Zhou, and Alexander S Aruin. TeagerKaiserenergy operation of surface EMG improves muscle activity onset detection. Annals of biomedical engineering, 35(9):15321538, 2007. https://doi.org/10.1007/s10439-007-9320-z

[13] Richard T Lauer and Laura A Prosser. Use of the TeagerKaiser energy operator for muscle activity detection in children. Annals of biomedical engineering, 37(8):1584-1593, 2009. https://doi.org/10.1007/s10439-009-9727-9

[14] Stanislaw Solnik, Patrick Rider, Ken Steinweg, Paul DeVita, and Tibor Hortobágyi. Teager-Kaiser energy operator signal conditioning improves EMG onset detection. European Journal of Applied Physiology, 110(3):489-498, 2010. https://doi.org/10.1007/s00421-010-1521-8

[15] Hichem Bengacemi, Karim Abed-Meraim, Olivier Buttelli, Abdelaziz Ouldali, and Ammar Mesloub. A new detection method for EMG activity monitoring. Medical \& Biological Engineering \& Computing, 58(2):319-334, 2020. https://doi.org/10.1007/s11517-019-02048-0

[16] Paolo Soda, Stefano Mazzoleni, Giuseppe Cavallo, Eugenio Guglielmelli, and Giulio Iannello. Human movement onset detection from isometric force and torque measurements: A supervised pattern recognition approach. Artificial intelligence in medicine, 50(1):55-61, 2010. https://doi.org/10.1016/j.artmed.2010.04.008
[17] Amal Naseem, Meryem Jabloun, Olivier Buttelli, and Phillippe Ravier. Detection of sEMG muscle activation intervals using gaussian mixture model and ant colony classifier. In 2016 24th European Signal Processing Conference (EUSIPCO), pages 1713-1717. IEEE, 2016. https://doi.org/10.1109/EUSIPCO.2016.7760541

[18] Olivier Buttelli. Agence nationale de la recherche. http://www.agence-nationale-recherche.fr/Projet-ANR-12TECS-0020, 2012.

[19] Dinesh D Patil, RP Singh, Vilas M Thakare, and Avinash K Gulve. Automatic classification of ECG arrhythmia using morphological parameters with HMM and SVM. International Journal of Applied Engineering Research, 12(20):10376-10384, 2017.

[20] Dihong Jiang, Ya-nan Lu, MA Yu, and WANG Yuanyuan. Robust sleep stage classification with single-channel EEG signals using multimodal decomposition and hmm-based refinement. Expert Systems with Applications, 121:188-203, 2019. https://doi.org/10.1016/j.eswa.2018.12.023

[21] Mohamed Nait-Meziane, Abdenour Hacine-Gharbi, Philippe Ravier, Guy Lamarque, Jean-Charles Le Bunetel, and Yves Raingeaud. HMM-based transient and steady-state current signals modeling for electrical appliances identification. In Proceedings of the 5th International Conference on Pattern Recognition Applications and Methods, pages 670-677. SCITEPRESSScience and Technology Publications, Lda, 2016.

[22] Abed Khorasani and Mohammad Reza Daliri. HMM for classification of parkinson's disease based on the raw gait data. Journal of medical systems, 38(12):147, 2014. https://doi.org/10.1007/s10916-014-0147-5

[23] Matteo Rossi, Simone Benatti, Elisabetta Farella, and Luca Benini. Hybrid EMG classifier based on HMM and SVM for hand gesture recognition in prosthetics. In 2015 IEEE International Conference on Industrial Technology (ICIT), pages 1700-1705. IEEE, 2015. https://doi.org/10.1109/ICIT.2015.7125342

[24] Jang-Woo Kwon, Jung-Ho Kim, and Heung-Ho Choi. Classification of the EMG signal using cascaded classifier. In World Congress on Medical Physics and Biomedical Engineering 2006, pages 1222-1225. Springer, 2007. https://doi.org/10.1007/978-3540-36841-0_295

[25] Adrian DC Chan, Kevin Englehart, Bernard Hudgins, and Dennis Fenton Lovely. Hidden markov model classification of myoelectric signals in speech. IEEE Engineering in Medicine and Biology Magazine, 21(5):143-146, 2002. https://doi.org/10.1109/MEMB.2002.1044184

[26] Steve Young, Gunnar Evermann, Mark Gales, Thomas Hain, Dan Kershaw, Xunying Liu, Gareth Moore, Julian Odell, Dave Ollason, Dan Povey, et al. The HTK book. Cambridge university engineering department, 3:175, 2002.

[27] Abdenour Hacine-Gharbi and Philippe Ravier. Wavelet cepstral coefficients for electrical appliances identification using hidden markov models. In ICPRAM, pages 541-549, https://doi.org/2018. 10.5220/0006662305410549

[28] V Shah, R Anstotz, I Obeid, and J Picone. Adapting an automatic speech recognition system to event classification of electroencephalograms. In 2018 IEEE Signal Processing in Medicine and Biology Symposium (SPMB), pages 1-5. IEEE, 2018. https://doi.org/10.1109/SPMB.2018.8615625

[29] Abdenour Hacine-Gharbi and Philippe Ravier. On the optimal number estimation of selected features using joint histogram based mutual information for speech emotion recognition. Journal of King Saud University-Computer and Information Sciences, 2019. https://doi.org/10.1016/j.jksuci.2019.07.008 
[30] Jean-Paul Haton, Christophe Cerisara, Dominique Fohr, Yves Laprie, and Kamel Smaïli. Reconnaissance automatique de la parole: Du Signal à son Interprétation. Dunod, 2006.

[31] Abdenour Hacine-Gharbi. Sélection de paramètres acoustiques pertinents pour la reconnaissance de la parole. $\mathrm{PhD}$ thesis, 2012.

[32] Claude C Chibelushi, Farzin Deravi, and John SD Mason. A review of speech-based bimodal recognition. IEEE transactions on multimedia, 4(1):23-37, 2002. https://doi.org/10.1109/6046.985551

[33] Dario Farina, Elena Fortunato, and Roberto Merletti. Noninvasive estimation of motor unit conduction velocity distribution using linear electrode arrays. IEEE Transactions on Biomedical Engineering, 47(3):380-388, 2000. https://doi.org/10.1109/10.827303

[34] Marco Barbero, Roberto Merletti, and Alberto Rainoldi. Atlas of muscle innervation zones: understanding surface electromyography and its applications. Springer Science \& Business Media, 2012.

[35] Jingwei Too, AR Abdullah, Norhashimah Mohd Saad, N Mohd Ali, and H Musa. A detail study of wavelet families for EMG pattern recognition. International Journal of Electrical and Computer Engineering (IJECE), 8(6):4221-4229, 2018.

[36] MS Hussain, Mamun Bin Ibne Reaz, Faisal Mohd-Yasin, and Muhammad I Ibrahimy. Electromyography signal analysis using wavelet transform and higher order statistics to determine muscle contraction. Expert Systems, 26(1):35-48, 2009. https://doi.org/10.1111/j.1468-0394.2008.00483.x

[37] K Mahaphonchaikul, D Sueaseenak, C Pintavirooj, M Sangworasil, and S Tungjitkusolmun. EMG signal feature extraction based on wavelet transform. In ECTI-CON2010: The 2010 ECTI International Confernce on Electrical Engineering/Electronics, Computer, Telecommunications and Information Technology, pages 327-331. IEEE, 2010.

[38] Angkoon Phinyomark, Chusak Limsakul, and Pornchai Phukpattaranont. An optimal wavelet function based on wavelet denoising for multifunction myoelectric control. In 2009 6th International Conference on Electrical Engineering/Electronics, Computer, Telecommunications and Information Technology, volume 2, pages 1098-1101. IEEE, 2009. https://doi.org/10.1109/ECTICON.2009.5137236

Hichem Bengacemi received the engineering degree in electrical engineering and Magister degree in advanced signal processing techniques from the Ecole Militaire Polytechnique (EMP) of Algiers, Algeria, in 2005 and 2013 respectively. He received his Cotutelle PhD degree from the EMP, Algeria and the university of Orlèans, France in June 2021. His research interests are pbiomedical signal processing and neuromuscular physiology.

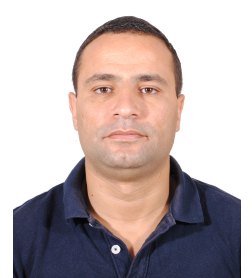

Abdenour Hacine-Gharbi received his Engineering and Magister degrees from University of Setif, Algeria in 1995 and 2002, respectively. He received his $\mathrm{PhD}$ from the University of Setif, Algeria and the University of Orlèans, France in 2012. $\mathrm{He}$ is currently an Assistant Professor at the Electronics Department of Bordj Bou Arreridj University, Algeria and member of LMSE Laboratory. His main research interests are pattern recognition, classification and feature selection.

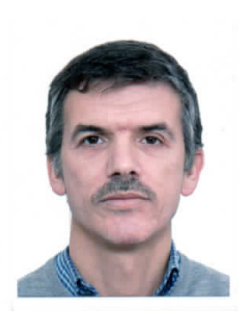

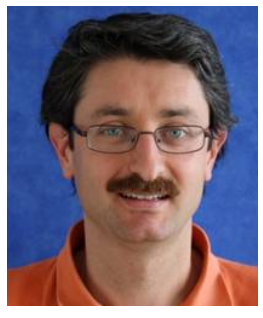

Philippe Ravier received the $\mathrm{Ph}$. D. degree in engineering science speciality signal, image and speech, from the Polytechnic National Institute of Grenoble (France) in 1998. Since 1999, he is assistant professor at Polytech Orlèans, the engineering school of Orlèans University, with habilitation to direct research obtained in 2011. His main researches deal with wavelet and time-frequency analysis with application to bioelectrical signals.

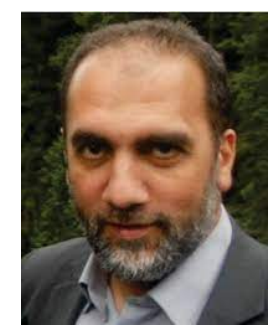

Karim Abed-Meraim was born in 1967. He received the State Engineering Degree from the Ecole Polytechnique, Palaiseau, France, in 1990, the State Engineering Degree from the Ecole Nationale Supèrieure des Tèlècommunications (ENST), Paris, France, in 1992, the M.Sc. degree from Paris XI University, Orsay, France, in 1992, and the Ph.D. degree in the field of signal processing and communications from ENST, in 1995. From 1995 to 1998, he was a Research Staff with the Electrical Engineering Department, The University of Melbourne, where he worked on several research project related to Blind System Identification for Wireless Communications, Blind Source Separation, and Array Processing for Communications. From 1998 to 2012, he has been an Assistant Professor, then an Associate Professor with the Signal and Image Processing Department, Tèlècom ParisTech. In September 2012, he joined the University of Orlèans, France (PRISME Laboratory), as a Full Professor. His research interests include signal processing for communications, adaptive filtering and tracking, array processing, and statistical performance analysis. He is the author of about 500 scientific publications, including book chapters, international journal and conference papers, and patents. He is an IEEE Fellow. Dr. Abed-Meraim is currently a member of the IEEE SAM-TC and a Senior Area Editor of the IEEE Transactions on Signal Processing.

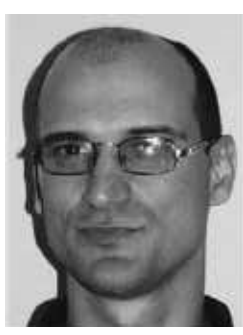

Olivier Buttelli was born in 1966. He receivedthe MS and Ph.D. degrees in biomechanics andmotor physiology in 1991 and 1996 both from the University of Paris 11 Orsay, respectively. He is associated professor at University of Orléans, specialized in biomechanics, physiology of muscle and ergonomics. His main activities relate to fatigue processes and physical exercise and development of signal processing for electrophysiological signals. 